\title{
Boundary shear flow past a cylinder near a wall
}

\author{
Lin Feng Chen ${ }^{\mathrm{a}}$, Guo Xiong $\mathrm{Wu}^{\mathrm{b}, *}$ \\ ${ }^{a}$ School of Naval Architecture and Ocean Engineering, Jiangsu University of Science and Technology, China \\ ${ }^{b}$ Department of Mechanical Engineering, University College London, London WC1E7JE, UK
}

\begin{abstract}
An investigation on boundary shear flow past a circular cylinder near a wall is numerically performed via a stabilized finite element method. The main focus is to uncover its major difference with the flows corresponding to the symmetry boundary, and to two identical circular cylinders in a side-by-side arrangement. In particular at Reynolds number $R e=100$, extensive simulations are made for different gaps between the cylinder and wall. It is noted that in the wake of the cylinder the vortex contour lines shift upwards. At $R e=100$, the flow behind a cylinder near the wall may be time dependent. With a reduction of the gap spacing to a magnitude in $(0.75,1)$, the vortex shedding nearly vanishes. For the flow behind two identical circular cylinders side and side, the flow may change from periodic flow to totally irregular one. The drag force $C_{D}$, lift force $C_{L, r m s}$ and Strouhal number $S t$ of the circular cylinder near the wall vary differently with the gap, compared with those in the other two cases. When the cylinder is located in the boundary layer, the boundary shear flow has strong effect on the hydrodynamic quantities. Extensive simulations are also made for $R e=200,400,600$ and 800 . It is found that the Reynolds number has strong effect on the flow and force on the cylinder, not only through the variation of $R e$ itself but also the boundary layer of the wall. Withe $R e$ increasing, strong vortex shedding from the near-wall cylinder at $e=0.5$ starts above a $R e$ in $(200,300)$.
\end{abstract}

Keywords: Boundary shear flow, flow past circular cylinder, stabilized finite element method, flow and force characteristics with wall effects

\section{Introduction}

Boundary shear flow past a cylinder near a wall, characterized by the mutual interaction between the vortices in the wake and the shear layers near the wall, has important engineering relevance, for example in the case of gas and oil pipelines and cables near the seabed. In such flow, the presence of the wall plays 5 an important role. From geometrical configuration point of view, the problem has similarity to (1) another cylinder which is placed at the mirror image position, with symmetry condition on the wall, (2) two cylinders in side-by-side arrangement (Figure 1). These cases may look similar in configuration. However, because of the difference in the conditions on the wall, their overall flow characteristics are completely different. Through numerical simulation, the present study investigates the flow past a single circular cylinder near a wall, and aims to uncover its major difference with the flows corresponding to the symmetry boundary, and to two identical circular cylinders in a side-by-side arrangement.

There has been a substantial volume of work on the flow past two circular cylinders in a side-by-side arrangement in the past few decades 1 , 2]. Typical work includes that by Williamson $\underline{3}$, who experimentally investigated the flow at $R e=50-200$ and $0.5<G \leq 5$ ( $G$ is the gap spacing between the two cylinder surfaces on the near side, which is nondimensionalized by the diameter of the cylinder $D$ ) using flowvisualization methods. It was found that vortex-shedding synchronization could occur either in phase or

\footnotetext{
*Corresponding author. Permanent address: Department of Mechanical Engineering, University College London, London WC1E7JE, UK

Email address: g.wu@ucl.ac.uk (Guo Xiong Wu)
} 


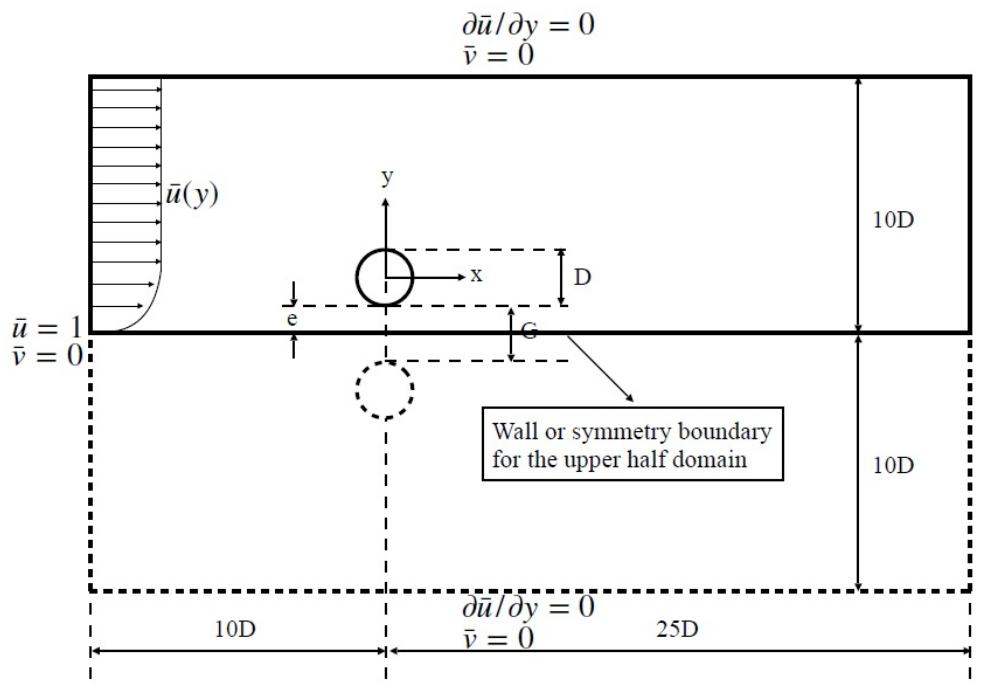

Figure 1: Schematic diagram of the present computational domain (only the upper half part is needed for cases with the wall and symmetry boundaries, for which $u=1$ is used at the inlet).

antiphase above a critical gap spacing. Below a certain gap spacing the flow became asymmetric or unsteady. Certain harmonic modes of vortex shedding were observed in the asymmetric regime whereby the shedding frequency on one side of the wake was a multiple of that on the other side. Kang [4] carried out a numerical 20 investigation on the problem using an immersed boundary method in a range of $40 \leq R e \leq 160$ and $G<5$. Six kinds of wake patterns: antiphase-synchronized, in-phase-synchronized, flip-flopping, deflected, single bluff-body and steady wake patterns were observed in the study. Experimental and numerical studies have also been performed in other ranges of physical parameters, such as those by Kim and Durbin [5], Xu et al. [6], Jiao and $\mathrm{Wu}[7]$. These studies have presented some in depth understanding of the underlying physics 25 of the complex flow past two cylinders.

The wake of two circular cylinders in a side-by-side arrangement is due to interaction of two rows of vortices shed from two cylinders respectively, and the intensity of interaction depends on the gap spacing between two cylinders. At $R e=100$, when $G>5$, the two cylinders become virtually independent and each of them can be treated in isolation. When $2<G<5$, the flow past two cylinders is similar to that 30 past a single cylinder with symmetry condition imposed on the symmetry line. The lift force coefficients of the two cylinders are in antiphase (or $180^{\circ}$ phase difference). At $0.4 \leq G \leq 1.5$, the wakes of two cylinder intersect with each other strongly, such that the vortex motion is no longer periodic and becomes irregular. For these cases, the flow characteristics are no longer similar to the flow with a symmetry boundary since the reflection of the symmetry boundary prevents the vertical velocity from such interaction in the wake.

In the case of the cylinder located in the vicinity of a wall, the incoming flow will be very much affected by the wall boundary layer, especially at relatively low Reynolds number. This means that the cylinder is in shear flow rather than in uniform flow. This may very much affect the features of flow past cylinder. Likewise, it is expected that the wall boundary layer may substantially affect the process of vortex shedding and its subsequent motion in the wake. Some studies on such a flow have been conducted (Bearman and

40 Zdravkovich [8, 9], Buresti et al. [10], Grass et al. 11], Taniguchi and Miyakoshi[12], Lei et al. [13], Price et al. [14], Wang and Tan[15, Taneda[16]). Most of those studies were carried out at Reynolds number in the sub-critical flow regime $\left(O\left(10^{4}\right)-O\left(10^{5}\right)\right)$. The flow regimes are relatively insensitive to the Reynolds number within this range. The wall boundary effect on the flow patterns, vorticities, vortex shedding and hydrodynamic quantities with different gap spacing between the cylinder bottom and the wall boundary 45 were investigated. It has been demonstrated that in sub-critical flow regimes the vortex shedding in the wake is suppressed when $e$ ( $e$ is the spacing between the cylinder bottom and the wall boundary which is nondimensionalized by $D$, namely, $e=G / 2$ ) is less than about $0.3[17$. Teneda[16] and Lei et al. [18] carried 
out both experimental and numerical studies at Reynolds numbers $(R e<1000)$. Some explanations for vortex shedding suppression at small gap were provided.

The objective of the present study is to undertake further in depth investigation into the flow past a cylinder near a wall. In particular, we will focus on the cases in which the boundary layer of the wall may have a stronger effect. Therefore the Reynolds numbers chosen are relatively low, in which the thickness of boundary layer may be comparable to the gap between the cylinder and the wall. We shall then compare the results with those for two cylinders in a side-by-side arrangement and a single cylinder near a symmetry

55 boundary, in both of which, the incoming flow is uniform. We then try to acquire some deeper understanding on how the shear layer of the wall and the wall itself will affect the vortex shedding process and its subsequent motion. The numerical method used in the present study is the stabilized finite element method [19, 20, 21. A generalized- $\alpha$ method of second order accuracy 22 is applied to temporal advance with a specified high temporal frequency damping, which eliminates non-physical instabilities.

In particular, we deal with the flows in a range of $100 \leq R e \leq 800$ and $G \leq 5$. Extensive results are provided to show the sensitivity of the flow to the gap spacing $e$ (or $G$ ), and how this compares with the flows in two cylinder case and single cylinder near the symmetry line case. We aim to uncover the underlying mechanism of the wake interaction in these three cases, and to investigate how the flow structures in the wake influence the hydrodynamic quantities. Investigations on the effects of the Reynolds number in these -5 cases are also performed and results are compared. Noticeably, the three-dimensional wake transition might appear for a single cylinder at Re above 200[23. On the other hand, the work by Prsic et al. 24] revealed that the mean drag force and root-mean-square(RMS) of lift force of the $2 \mathrm{D}$ and $3 \mathrm{D}$ simulations differed by less than $1 \%$ at $R e=100$ and 500 . Only when $R e \geq 1000$, some of results from the $2 \mathrm{D}$ simulations started to deviate notably from those from 3D. Considering the main purpose of the present study is on the effect of the wall and boundary layer through comparison with results from similar configurations, the 3D effect is not expected to have major influence on the main focus of this paper.

The paper is organized as follows. The finite element method, generalized- $\alpha$ method and problem setup are provided in Section 2. Validation, results and discussion on the effect of the gap to diameter ratio as well as the Reynolds number are presented in Section 3. Finally, conclusions are given in Section 4.

\section{2. Mathematical formulation}

\subsection{Governing equation}

The two dimensional (2D) continuity equation and Navier-Stokes equations for the incompressible viscous flows are used. The non-dimensional forms of these equations, based on the incoming velocity $U_{c}$ of the flow, body dimension $L$ and kinetic viscosity $\nu$ of the fluid, can be written as

$$
\begin{aligned}
\nabla \cdot \boldsymbol{u} & =0, \\
\mathcal{L}(\boldsymbol{u}, p)=\frac{\partial \boldsymbol{u}}{\partial t}+(\boldsymbol{u} \cdot \nabla) \boldsymbol{u}+\nabla p-\frac{1}{R e} \nabla^{2} \boldsymbol{u} & =0,
\end{aligned}
$$

where $\boldsymbol{u}$ denotes the velocity, $p$ denotes the pressure, $R e=U_{c} L / \nu$ is Reynolds number.

\subsection{Discrete formulation}

The numerical method used in the study is based on a finite element method. Multiplying Eqs. (1) and (2) by the weighting function $\boldsymbol{W}=\{q, \boldsymbol{w}\}$, respectively, and integrating the result over the fluid domain, we have their standard Galerkin formulations

$$
\begin{aligned}
(q, \nabla \cdot \boldsymbol{u})_{\Omega} & =0 \\
\left(\boldsymbol{w}, \frac{\partial \boldsymbol{u}}{\partial t}\right)_{\Omega}+(\boldsymbol{w}, \boldsymbol{u} \cdot \nabla \boldsymbol{u})_{\Omega}+(\boldsymbol{w}, \nabla p)_{\Omega}-\left(\boldsymbol{w}, \frac{1}{R e} \nabla^{2} \boldsymbol{u}\right)_{\Omega} & =0,
\end{aligned}
$$

where $\Omega$ denotes the fluid domain, $(,)_{\Omega}$ represents an integration in the space domain. In this study, bilinear shape and weighting functions are used for the above discrete formulation. 
It is often that the standard Galerkin formulations (3) and (4) may become ill-conditioned and the solution procedure may become unstable, especially when the linear shape function is used, which is known as the LBB condition. In order to circumvent the instability, we use overbar to indicate $\boldsymbol{u}$ and $p$ in Eqs. (3) and (4), and then modify these two equations as

$$
\begin{array}{r}
(q, \nabla \cdot \overline{\boldsymbol{u}})_{\Omega}+\left(q, \nabla \cdot \boldsymbol{u}^{\prime}\right)_{\Omega}=0 \\
\left(\boldsymbol{w}, \frac{\partial \overline{\boldsymbol{u}}}{\partial t}\right)_{\Omega}+(\boldsymbol{w}, \overline{\boldsymbol{u}} \cdot \nabla \overline{\boldsymbol{u}})_{\Omega}+\left(\boldsymbol{w}, \overline{\boldsymbol{u}} \cdot \nabla \boldsymbol{u}^{\prime}\right)_{\Omega}+(\boldsymbol{w}, \nabla \bar{p})_{\Omega} \\
+\left(\boldsymbol{w}, \nabla p^{\prime}\right)_{\Omega}-\left(\boldsymbol{w}, \frac{1}{R e} \nabla^{2} \overline{\boldsymbol{u}}\right)_{\Omega}=0
\end{array}
$$

where

$$
\begin{aligned}
& \boldsymbol{u}^{\prime}=-\boldsymbol{\tau}_{m} \mathcal{L}(\overline{\boldsymbol{u}}, p), \\
& p^{\prime}=-\tau_{c} \nabla \cdot \overline{\boldsymbol{u}}
\end{aligned}
$$

where $\mathcal{L}(\overline{\boldsymbol{u}}, p)$ is the residual of Eq. (2). In the above two equations, $\boldsymbol{\tau}_{m}$ and $\tau_{c}$ are defined as follows 25, 26]

$$
\begin{aligned}
\tau_{m} & =\left(c_{1} \overline{\boldsymbol{u}} \boldsymbol{G} \overline{\boldsymbol{u}}+\frac{c_{2}}{R e^{2}} \boldsymbol{G}: \boldsymbol{G}+\frac{c_{3}}{\Delta t^{2}}\right)^{-\frac{1}{2}} \\
\tau_{c} & =\frac{\overline{\boldsymbol{u}} \boldsymbol{G} \overline{\boldsymbol{u}}}{\operatorname{tr}(\boldsymbol{G})}
\end{aligned}
$$

where : denotes a double dot product, $c_{1}, c_{2}$ and $c_{3}$ are constants depending on the element type, which, for bilinear shape functions used in this paper, are taken as $c_{1}=1, c_{2}=36$ and $c_{3}=4$. $\boldsymbol{G}$ is a covariant metric tensor of the gradient of local element spatial coordinates $\xi$ with respect to the global coordinates $x$ for the same point

$$
\boldsymbol{G}=\left(\frac{\partial \boldsymbol{\xi}}{\partial \boldsymbol{x}}\right)^{T} \frac{\partial \boldsymbol{\xi}}{\partial \boldsymbol{x}},
$$

and $\frac{\partial \boldsymbol{\xi}}{\partial \boldsymbol{x}}$ is the Jacobian matrix. $\operatorname{tr}(\boldsymbol{G})$ in Eq. 10 represents a summation of the diagonal elements of $\boldsymbol{G}$.

To avoid the presence of the derivative of $\boldsymbol{u}$ and $p^{\prime}$, as well as the second derivative of $\overline{\boldsymbol{u}}$, the terms involved with $\nabla \boldsymbol{u}^{\prime}, \nabla p^{\prime}$ and $\nabla^{2} \overline{\boldsymbol{u}}$ are integrated by parts. Ignoring the effects of $\boldsymbol{u}^{\prime}$ and $p^{\prime}$ on the boundary, Eqs. (3) and (4) become

$$
\begin{array}{r}
(q, \nabla \cdot \overline{\boldsymbol{u}})_{\Omega}-\left(\nabla q, \boldsymbol{u}^{\prime}\right)_{\Omega}=0 \\
\left(\boldsymbol{w}, \frac{\partial \overline{\boldsymbol{u}}}{\partial t}\right)_{\Omega}-\left(\nabla \boldsymbol{w}, \overline{\boldsymbol{u} \otimes \overline{\boldsymbol{u}})_{\Omega}}+(\boldsymbol{w}, \nabla \bar{p})_{\Omega}+\left(\nabla \boldsymbol{w}, \frac{1}{R e} \nabla \overline{\boldsymbol{u}}\right)_{\Omega}\right. \\
-\left(\nabla \boldsymbol{w}, \overline{\boldsymbol{u}} \otimes \boldsymbol{u}^{\prime}\right)_{\Omega}-\left(\nabla \cdot \boldsymbol{w}, p^{\prime}\right)_{\Omega} \\
+\left(\boldsymbol{w},(\overline{\boldsymbol{u}} \cdot \boldsymbol{n}) \overline{\boldsymbol{u}}-\frac{1}{R e} \nabla \overline{\boldsymbol{u}} \cdot \boldsymbol{n}\right)_{\Gamma}=0 .
\end{array}
$$

where $\otimes$ denotes the tensor product, or $\overline{\boldsymbol{u}} \otimes \overline{\boldsymbol{u}}=\bar{u}_{i} \bar{u}_{j}, \Gamma$ represents the fluid domain boundary, and $\boldsymbol{n}$ denotes the outward normal vector to the fluid domain boundary.

\subsection{Time discretization}

In the present study, we employ a generalized- $\alpha$ method, which was applied to fluid dynamics in [22, 27, 28, to solve Eqs. (12) and (13). Define $\boldsymbol{a}$ as the nodal values of $\{\boldsymbol{u}, p\}^{T}, \dot{\boldsymbol{a}}$ are the temporal derivative of $\boldsymbol{a}$ at the same nodes. Performing integrations in Eqs. (12) and 13 results in a system of nonlinear ordinary differential equations

$$
A \dot{\boldsymbol{a}}=B \boldsymbol{a} .
$$


Introducing the generalized- $\alpha$ method for the above system yields

$$
\begin{aligned}
& \boldsymbol{R}\left(\dot{\boldsymbol{a}}_{n+\alpha_{m}}, \boldsymbol{a}_{n+\alpha_{f}}\right)=A \dot{\boldsymbol{a}}_{n+\alpha_{m}}-B \boldsymbol{a}_{n+\alpha_{f}}=\mathbf{0}, \\
& \boldsymbol{a}_{n+1}=\boldsymbol{a}_{n}+\Delta t \dot{\boldsymbol{a}}_{n}+\gamma \Delta t\left(\dot{\boldsymbol{a}}_{n+1}-\dot{\boldsymbol{a}}_{n}\right), \\
& \dot{\boldsymbol{a}}_{n+\alpha_{m}}=\dot{\boldsymbol{a}}_{n}+\alpha_{m}\left(\dot{\boldsymbol{a}}_{n+1}-\dot{\boldsymbol{a}}_{n}\right), \\
& \boldsymbol{a}_{n+\alpha_{f}}=\boldsymbol{a}_{n}+\alpha_{f}\left(\boldsymbol{a}_{n+1}-\boldsymbol{a}_{n}\right),
\end{aligned}
$$

where $\Delta t=t_{n+1}-t_{n}$ is the time step size which is taken as a constant in the present work, $\alpha_{m}$ and $\alpha_{f}$ are parametric constants with

$$
\alpha_{m}=\frac{1}{2}\left(\frac{3-\zeta}{1+\zeta}\right), \quad \alpha_{f}=\frac{1}{1+\zeta},
$$

$\zeta$ is set to 0.5 to retain unconditional stability and a second-order accuracy[22], and $\gamma=0.5+\alpha_{m}+\alpha_{f}$.

Here a prediction-multicorrection procedure is used to handle the above generalized- $\alpha$ method algorithms. The procedure starts with a prediction of the solution and its temporal derivative at $t_{n+1}$

$$
\begin{aligned}
\boldsymbol{a}_{n+1}^{(0)} & =\boldsymbol{a}_{n}, \\
\dot{\boldsymbol{a}}_{n+1}^{(0)} & =\frac{\gamma-1}{\gamma} \dot{\boldsymbol{a}}_{n} .
\end{aligned}
$$

Substituting these predictions into Eqs. (17) and (18), and the results into Eq (15), we can force $\boldsymbol{R}=0$ by correction of $\boldsymbol{a}_{n+\alpha_{f}}^{(i)}$ through $\Delta \boldsymbol{a}_{n+\alpha_{f}}^{(i)}$. Utilizing a Newton's iteration procedure we have

$$
\boldsymbol{R}\left(\dot{\boldsymbol{a}}_{n+\alpha_{m}}^{(i)}, \boldsymbol{a}_{n+\alpha_{f}}^{(i)}\right)+\frac{\partial \boldsymbol{R}\left(\dot{\boldsymbol{a}}_{n+\alpha_{m}}^{(i)}, \boldsymbol{a}_{n+\alpha_{f}}^{(i)}\right)}{\partial \boldsymbol{a}_{n+\alpha_{f}}^{(i)}} \Delta \boldsymbol{a}_{n+\alpha_{f}}^{(i)}=0
$$

Once $\Delta \boldsymbol{a}_{n+\alpha_{f}}^{(i)}$ is obtained, $\boldsymbol{a}_{n+\alpha_{f}}^{(i)}$ and $\dot{\boldsymbol{a}}_{n+\alpha_{m}}^{(i)}$ are updated via

$$
\begin{aligned}
\boldsymbol{a}_{n+\alpha_{f}}^{(i+1)} & =\boldsymbol{a}_{n+\alpha_{f}}^{(i)}+\Delta \boldsymbol{a}_{n+\alpha_{f}}^{(i)}, \\
\dot{\boldsymbol{a}}_{n+\alpha_{m}}^{(i+1)} & =\left(1-\frac{\alpha_{m}}{\gamma}\right) \dot{\boldsymbol{a}}_{n}+\frac{\alpha_{m}}{\gamma \Delta_{t} \alpha_{f}}\left(\boldsymbol{a}_{n+\alpha_{f}}^{(i+1)}-\boldsymbol{a}_{n}\right) .
\end{aligned}
$$

Here Eq. (24) is obtained from Eqs. (16)-(18) by eliminating $\boldsymbol{a}_{n+1}$ and $\dot{\boldsymbol{a}}_{n+1}$. This process continues utill $\Delta \boldsymbol{a}_{n+\alpha_{f}}^{(i)}$ is less than a given tolerance. With the solved $\boldsymbol{a}_{n+\alpha_{f}}^{(i)}$ and $\dot{\boldsymbol{a}}_{n+\alpha_{m}}^{(i)}$, the solutions at $t_{n+1}$ are determined using Eqs. (17) and (18), and computation moves to the next time step.

\subsection{Problem setup}

Simulations through the above described procedure are performed for a circular cylinder of diameter $D$ in a rectangular computational domain (Figure 1). The size of the whole computational domain is $35 D$ by $20 \mathrm{D}$. The centre of the cylinder is located at the origin of the coordinate system $\left(x_{c}=0, y_{c}=0\right)$, which is $10 D$ downstream the flow inlet, and the flow outlet is located $25 D$ downstream the centre of the cylinder. These distances are comparable to those in previous publications, for example in $\mathrm{Wu} \& \mathrm{Hu}(2008)[29$ and are sufficient to neglect the effects of the boundary truncation far upstream and downstream of the cylinder. For the cases with the wall and symmetry boundaries, the top boundary is located at a height of $10 D$ from the centre of the cylinder. This ensures that it has negligible effect on the flow past the cylinder.

In all the numerical simulations, the characteristic length $L=D$ and no-slip boundary conditions are applied to the cylinder surface as well as the bottom wall. However the impermeable condition is applied on the latter when symmetry boundary is used. At the flow inlet, a constant velocity value is given for the 
two-cylinder and symmetry boundary cases. For the wall boundary case, an approximate sheared profile based on the Blasius laminar boundary layer theory is prescribed at the inlet,

$$
\bar{u}(y)= \begin{cases}2 \frac{(y+e+0.5)}{\delta}-\frac{(y+e+0.5)^{2}}{\delta^{2}} & \text { if }(y+e+0.5) \leq \delta \\ 1 & \text { otherwise }\end{cases}
$$

where $y$ is perpendicular to the wall and $y=-e-0.5$ is the wall surface,

$$
\delta=4.92 \sqrt{\frac{\left(x-x_{s}\right)}{R e}}
$$

denotes the boundary layer thickness 30 and $x_{s}$ is the starting point of the boundary layer. In the simulations below, $\delta$ is set as a fixed value of 1.733 at the inlet. Thus at different $R e, x_{s}$ is adjusted accordingly. Correspondingly, at different $R e$, the boundary layer thickness at $x=0$ where the cylinder centre is located is given in Table 1). For the top side of the domain, a boundary condition of $\left(\frac{\partial \bar{u}}{\partial y}=0, \bar{v}=0\right)$ is used. Thus

Table 1: Boundary layer thickness at the cylinder location for different Reynolds numbers.

\begin{tabular}{ccccccc}
\hline Reynolds number $(R e)$ & 100 & 200 & 300 & 400 & 600 & 800 \\
\hline Boundary layer thickness $(\delta)$ & 2.329 & 2.05 & 1.95 & 1.899 & 1.845 & 1.8177 \\
\hline
\end{tabular}
condition (NC) in which the normal derivative of the velocity is prescribed, is imposed. In the former, DC is used explicitly on the boundary in the finite element formation in Eqs.12) and (13), and those $\boldsymbol{w}$ corresponding to the nodes on the boundary are not included in the weight process of Galerkin method. In the latter, $\mathrm{NC}$ is applied directly in the finite element formulation in $\mathrm{Eq}(13)$. At the flow outlet the following outflow boundary conditions [27] is employed

$$
-\left(\{\overline{\boldsymbol{u}} \cdot \boldsymbol{n}\}_{-}\right) \overline{\boldsymbol{u}}+\frac{1}{R e} \nabla \overline{\boldsymbol{u}} \cdot \boldsymbol{n}=\mathbf{0},
$$

where the term $\{\overline{\boldsymbol{u}} \cdot \boldsymbol{n}\}_{\text {_ }}$ denotes the negative part of $\overline{\boldsymbol{u}} \cdot \boldsymbol{n}$, that is

$$
\begin{cases}\{\overline{\boldsymbol{u}} \cdot \boldsymbol{n}\}_{-}=\overline{\boldsymbol{u}} \cdot \boldsymbol{n} & \text { if } \overline{\boldsymbol{u}} \cdot \boldsymbol{n}<0 \\ \{\overline{\boldsymbol{u}} \cdot \boldsymbol{n}\}_{-}=0 & \text { otherwise }\end{cases}
$$

The first term on the left-hand-side in $(26)$ is non zero only in the case of reverse flow through the outlet.

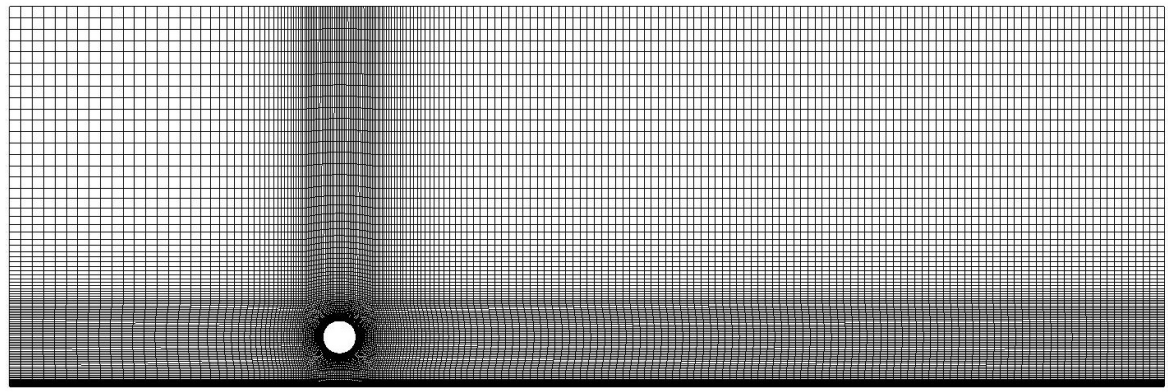

(a)

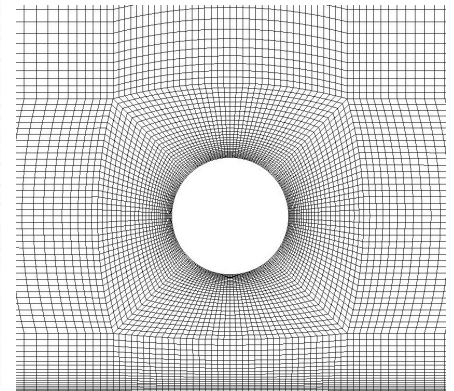

(b)

Figure 2: Mesh for a cylinder near the wall $(e=1)$. (a) whole computational domain, (b) near the cylinder.

Substituting Eq. (26) into Eq. (13) on the outlet boundary, the contribution from this boundary will then contain only

$$
+\int_{\Gamma_{\text {outlet }}} \boldsymbol{w}\left(\{\overline{\boldsymbol{u}} \cdot \boldsymbol{n}\}_{+}\right) \overline{\boldsymbol{u}} d \Gamma_{\text {outlet }}
$$


where

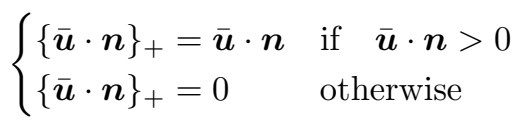

In the study, a body-fitted, structured multi block mesh of quadrilateral elements is used, which is generated by ICEM CFD. A sketch of the mesh for the case $e=1$ is illustrated in Figure 2 For all the simulations, the size of the elements near the cylinder and the wall boundary is set such that $\eta^{+}=u_{\tau} \eta R e<1$, where $u_{\tau}$ denotes the friction velocity at the wall, and $\eta$ denotes the element thickness in the normal direction to the wall.

\section{Results and discussion}

To validate the present numerical method and computer code, flow past a single circular cylinder at $R e=100$ is first simulated. In the computation, a mesh with 26020 elements, which is at the same level as that of other cases used below is adopted. A temporal step of $\Delta t=0.05$ is used for time advancement. A

Table 2: Hydrodynamic force coefficients of a single circular cylinder at $R e=100$.

\begin{tabular}{ccccc}
\hline \hline & $\bar{C}_{D}$ & $C_{L, \max }$ & $C_{L, r m s}$ & $S t$ \\
\hline Present & 1.3418 & 0.3375 & 0.237 & 0.166 \\
Park et al.[31] & 1.33 & 0.3321 & & 0.165 \\
Kang[4] & 1.33 & 0.32 & & 0.165 \\
Placzek et al. [32] & 1.37 & 0.33 & 0.23 & \\
\hline \hline
\end{tabular}
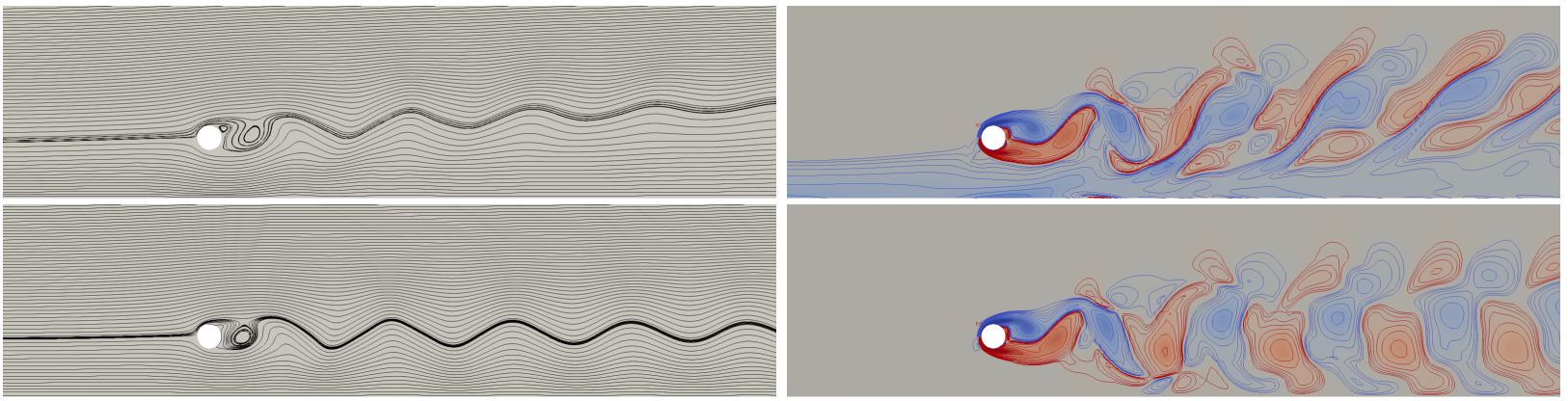

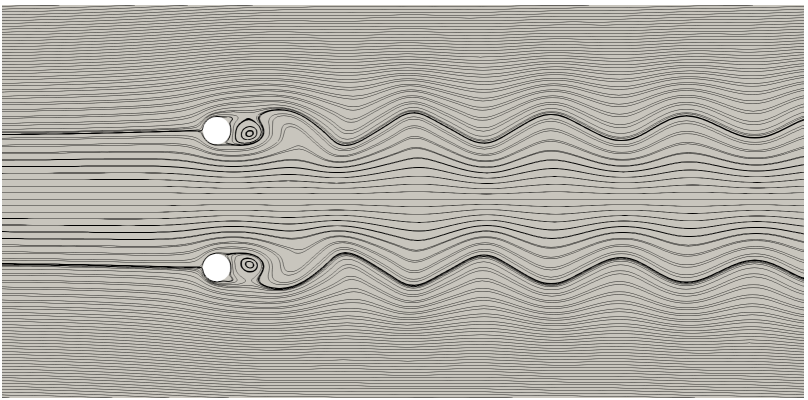

(a)

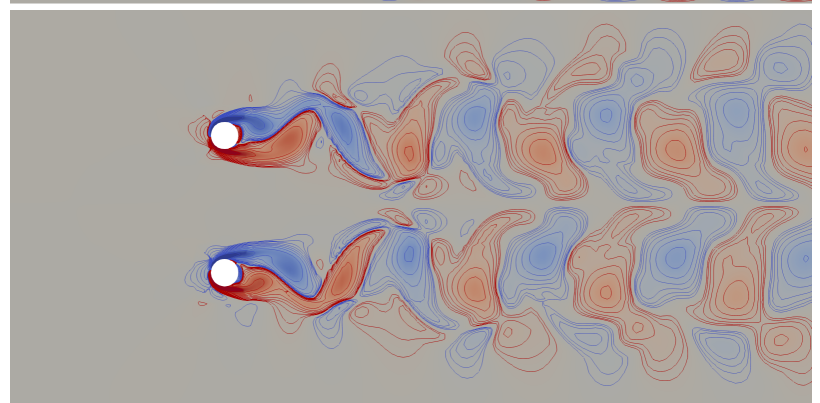

(b)

Figure 3: (a) Streamlines of instantaneous flow, (b) vorticity contours, red colour: positive vorticity, blue colour: negative vorticity, lines correspond to constant $w$ with $|w|<4$. From top to bottom: flow with a wall boundary $(e=2)$, symmetry boundary $(e=2)$ and flow past two cylinders $(G=4)$ at $R e=100$.

higher number of elements and a smaller $\Delta t$ have been used, and convergence has been achieved. Here, the 
typical results, including mean drag force coefficient $\left(\bar{C}_{D}\right)$, maximum of lift force $\left(C_{L, \max }\right)$, root-mean-square (RMS) of lift force

$$
C_{L, r m s}=\sqrt{\frac{1}{T_{2}-T_{1}} \int_{T_{1}}^{T_{2}}\left(C_{L}-\bar{C}_{L}\right)^{2} d t},
$$

and Strouhal number $(S t)$, are presented in Table 2. All these results are taken after the flow is fully developed and a periodic state is reached. Through the data in the table, it can be seen that all the typical results are in a good agreement with those obtained by others.

\subsection{Effect of the gap spacing}

As discussed in the introduction, when $G>5$, the mutual interference between two cylinder wakes is weak. As $G<5$ or $e<2.5$, the interference among multiple shear layers behind the cylinders affects strongly the flow in the wake, and the flow is very sensitive to the gap spacing. In the subsequent cases considered in this section, flow patterns and hydrodynamic quantities at $R e=100$ for $e<2.5$ with the wall and symmetry boundaries and the flow past two cylinders for $G<5$ are comparatively studied, using the obtained results when applicable.

\subsection{1. flow patterns}

The nondimensionalized vorticity

$$
w=\frac{\partial \bar{v}}{\partial x}-\frac{\partial \bar{u}}{\partial y}
$$

is introduced for the subsequent discussion. In Figure $3 \mathrm{a}$, at a given instant, streamlines are shown in line for $e=2$ or $G=4$, while the vorticity contour is shown in Figure 3b. It can be seen through the streamline patterns and vorticity contours that vortex shedding takes place in the three flow configurations. The flows
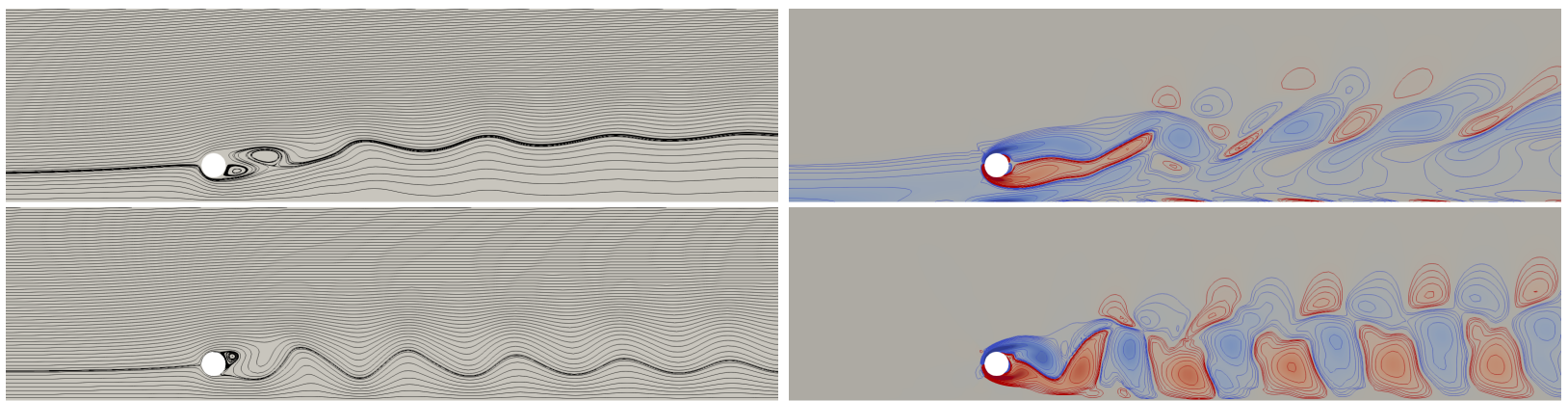

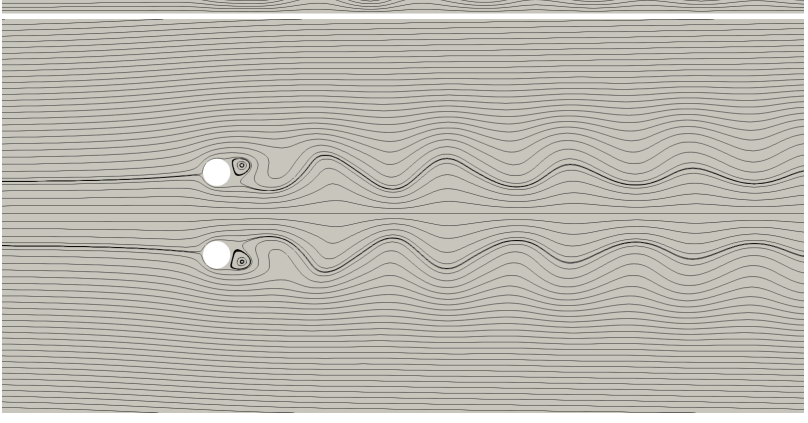

(a)

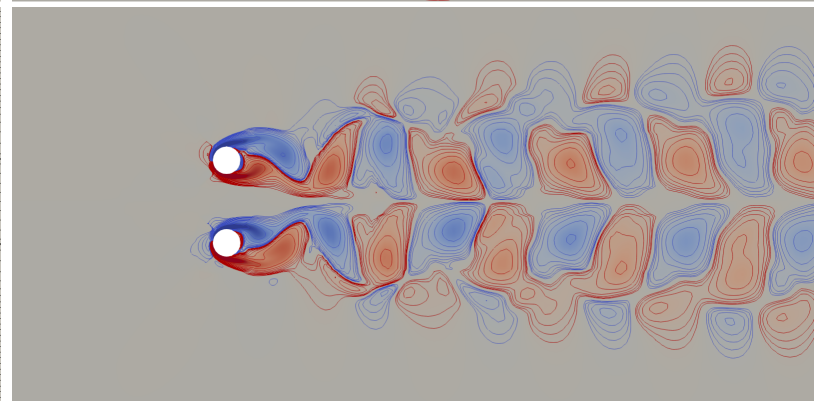

(b)

Figure 4: (a) Streamlines of instantaneous flow, (b) vorticity contours, red colour: positive vorticity, blue colour: negative vorticity, lines correspond to constant $w$ with $|w|<4$. From top to bottom: flow with a wall boundary $(e=1)$, symmetry boundary $(e=1)$ and flow past two cylinders $(G=2)$ at $R e=100$.

behind two cylinders is symmetric with respect to the centerline and antiphase-synchronized. The flow 
patterns and vorticity contour with the symmetry boundary are very similar to the upper half part of the flow past two cylinders. For the flow with the wall boundary, it is clearly visible that the shear layers from the cylinder interact with the shear layer from the wall boundary layer. One of the results is that it causes a vortex contour line shifting upwards.

As $e$ is reduced to $1(G=2)$, the flow past two cylinders still stays symmetric about the centerline and antiphase-synchronized, and its upper half is still similar to the flow with the symmetry boundary (shown in Figure 4). For the flow with the wall boundary, according to the streamlines, the flow velocity through the gap becomes low since the whole gap stays inside the boundary layer $(e=1<\delta=2.329)$. In fact the centre of the cylinder itself is also already in the boundary layer $(e+0.5=1.5<\delta=2.329)$. Therefore only the weak vortex shedding takes place in the wake in this case. From the vorticity contour, it can be observed that the interaction between the two shear layers behind the cylinder becomes less strong because of the weak shear layers due to the lower velocity through the gap, and the vortex contour pattern appears in a longer shape as a result of the effect of the shear layer near the wall.
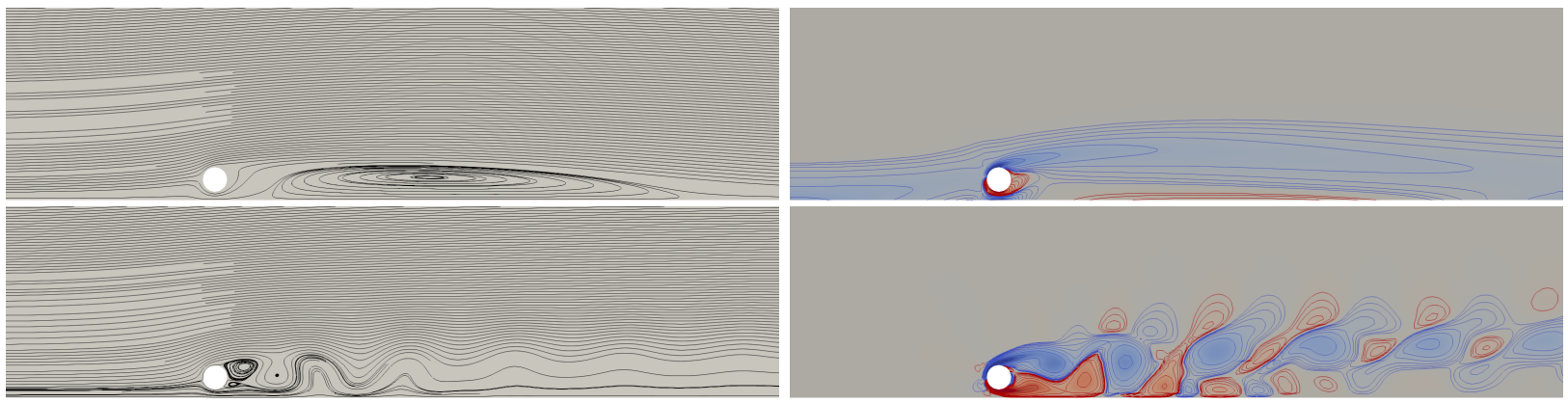

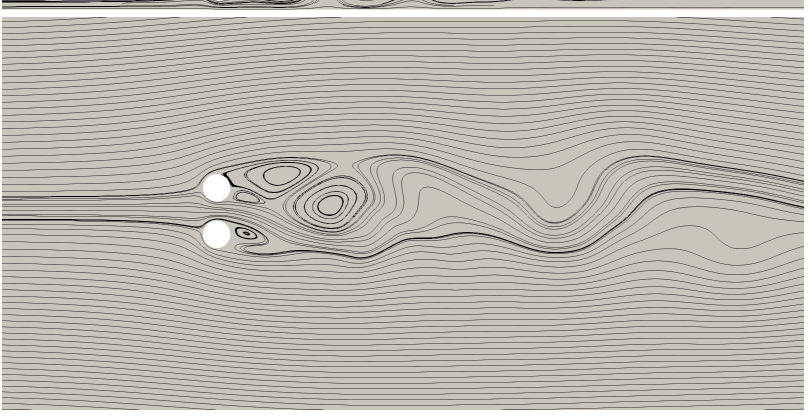

(a)

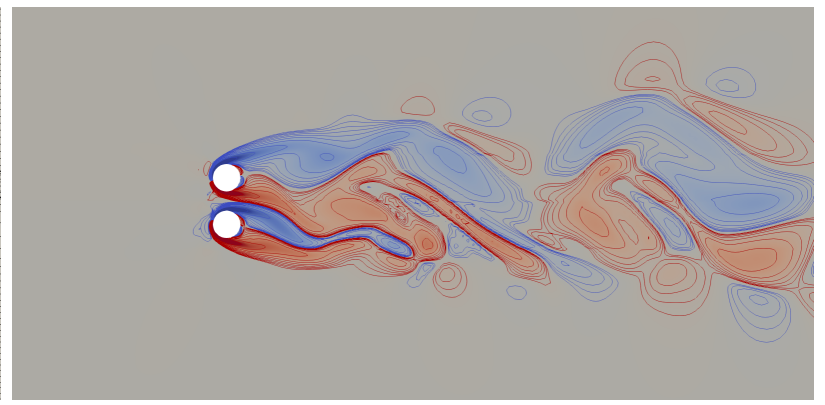

(b)

Figure 5: (a) Streamlines of instantaneous flow, (b) vorticity contours, red colour: positive vorticity, blue colour: negative vorticity, lines correspond to constant $w$ with $|w|<4$. From top to bottom: flow with a wall boundary $(e=0.35)$, symmetry boundary $(e=0.35)$ and flow past two cylinders $(G=0.7)$ at $R e=100$.

With further reduction of the gap spacing ( $e=0.35$ or $G=0.7$ ), the flow past two cylinders is no longer periodic (shown in Figure 5) when it is fully developed. The two downstream wakes mix together and becomes fully transient, which can be confirmed by the time evolution of lift force subsequently. The flow with the symmetry boundary still stays periodic. In the flow with the wall boundary, the gap is much smaller and the whole body is inside the boundary layer $(e+1=1.35<2.329)$. The wake eventually becomes steady with no strong vortex shedding.

For the flow with the wall boundary, it may be of interest to see the incoming flow to the cylinder along the vertical line passing through its left tip. We define an effective Reynolds number as

$$
R e_{e f}=u_{D} * R e,
$$

where

$$
u_{D}=\int_{-0.5}^{0.5} \bar{u}\left(x_{c}-0.5, y\right) d y
$$




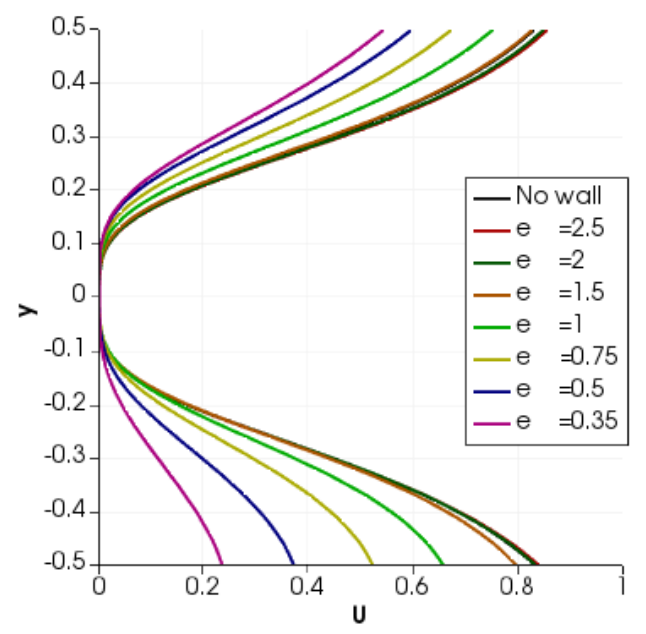

Figure 6: Mean horizontal velocity profiles of the flows with the wall boundary at $x=x_{c}-0.5$, over $-0.5 \leq y \leq 0.5$.

Table 3: Effective Reynolds numbers for flow past a circular cylinder close to the wall boundary at different gaps.

\begin{tabular}{ccccccccc}
\hline$e$ & No wall & 2.5 & 2 & 1.5 & 1 & 0.75 & 0.5 & 0.35 \\
\hline$R e_{e f}$ & 35.15 & 35.924 & 35.727 & 34.59 & 29.6 & 24.4 & 18.88 & 14.527 \\
\hline
\end{tabular}

Figure 6 shows the mean horizontal velocity profiles averaged after the flow reaches a periodic state at $x=x_{c}-0.5$, over $-0.5 \leq y \leq 0.5$. Here, the profile (black line) corresponding to uniform incoming flow past a single isolated cylinder is plotted for comparison. It can be seen that for the cases with a larger gap, for example, from $e=2.5$ to $e=1.5$, the velocity is slightly larger than the one of the single isolated cylinder. As the gap decreases, the velocity also deceases, especially over the lower part as it moves into the boundary layer. Based on the definition in Eq.(32), the effective Reynolds numbers for the different gap can be given in Table 3 When $e>=1.5$, the effective Reynolds number is almost the same as that a single cylinder in isolation. When $e<=1, R e_{e f}$ decreases rapidly with $e$. For $e=0.5$ and $0.35, R e_{e f}$ becomes much smaller than that for the single isolated cylinder. Correspondingly the flows in these cases are steady, as discussed previously.

\subsubsection{Hydrodynamic forces}

The hydrodynamic force coefficients of the cylinder for the three configurations are investigated in this section. The time evolution of the drag and lift force coefficients for three different $e$ or $G$ are shown in Figure 7. The results for the single isolated cylinder are also plotted in the figure as a reference. For $e=2$ or $G=4$, there is no major difference in the oscillation amplitudes of the drag force, the cylinder near the wall has the smallest drag force magnitude. The frequencies of the lift force are also similar, although there is some difference in magnitude. It can clearly be seen that the oscillation amplitude of the lift force of the cylinder in the boundary layer approaches that of the single isolated cylinder, and is lower than those in the other two cases. From $e=2$ ( or $G=4$ ) to $e=1$ ( or $G=2$ ), the drag force magnitude of the cylinder near the wall decreases evidently, and the oscillation amplitudes of the drag and lift forces decline drastically. The drag forces of the cylinder near the symmetry boundary and the upper cylinder slightly increase, and the oscillation amplitudes of the drag and lift force in those cases increase midly. As the gap spacing $e$ (or $G$ ) reduces to 0.35 (or 0.7), the oscillations of the drag and lift forces of the cylinder near the wall disappear, which is associated with the flow characteristics described previously. At this gap, the drag and lift forces 

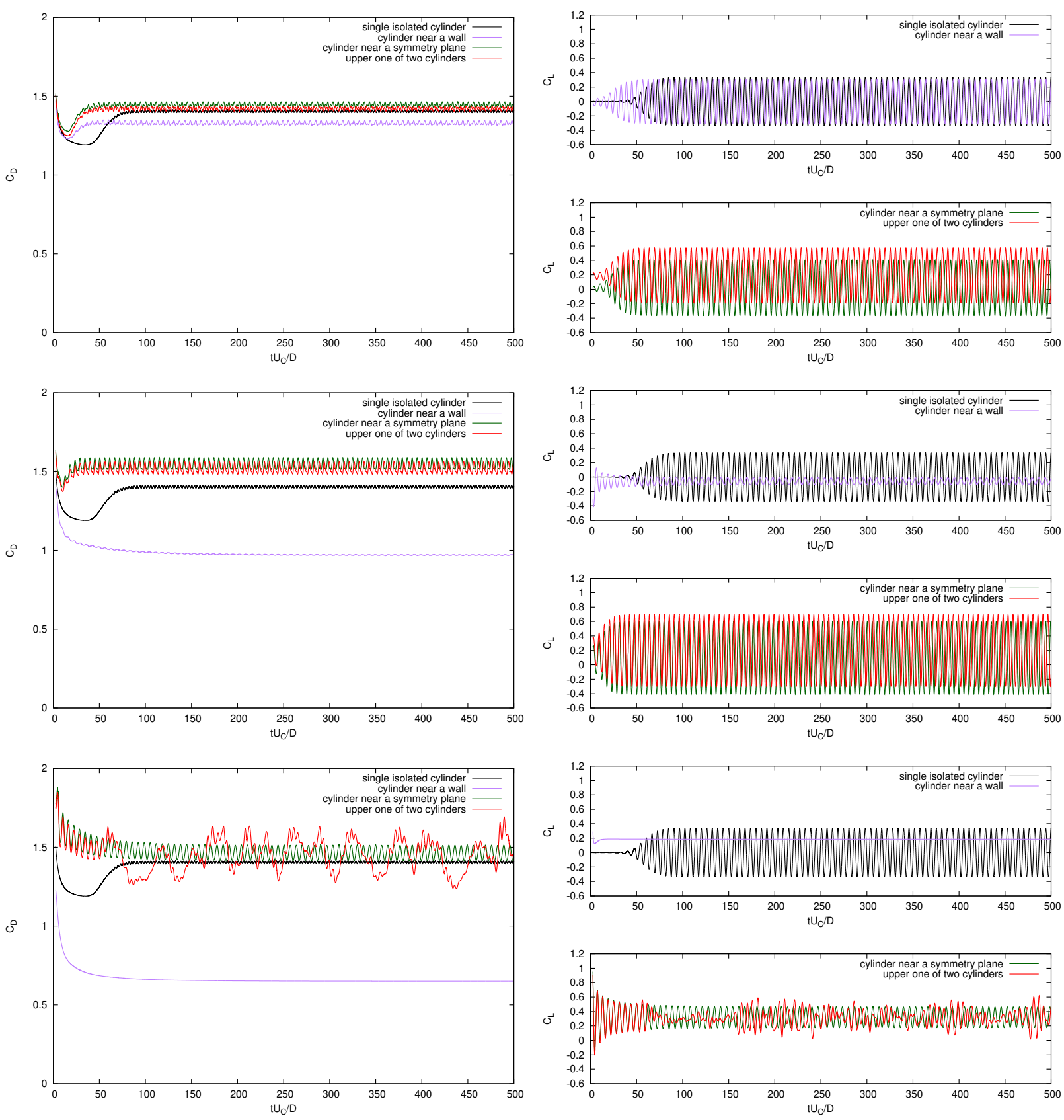

Figure 7: Variations of the drag $\left(C_{D}\right.$ and lift $\left(C_{L}\right)$ force coefficients with time for $e=2,1,0.35$ or $G=4$, $2,0.7$ (from top to bottom) at $R e=100$. 
two cylinders. The force coefficients of the cylinder with the symmetry boundary still remain periodic, and the oscillation amplitude of the lift force decreases.
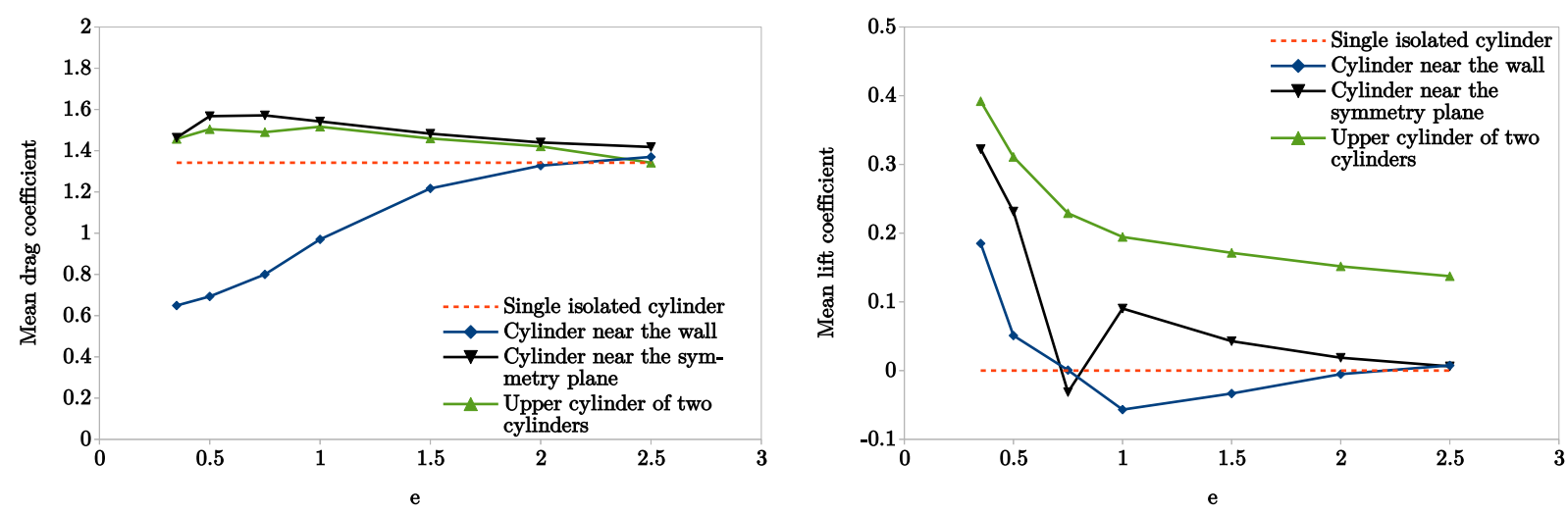

Figure 8: Variation of the mean $\operatorname{drag}\left(\bar{C}_{D}\right)$ and lift $\left(\bar{C}_{L}\right)$ force coefficients with the gap $e$ (or $\left.G\right)$ at $R e=100$.

The variations of the mean drag $\left(\bar{C}_{D}\right.$ and lift $\left(\bar{C}_{L}\right)$ force coefficients with the gap spacing are plotted in Figure 8. In the figure, the mean drag and lift force coefficients of a single isolated cylinder are shown in dashed line. The drag forces of the upper circular cylinder in the two cylinder case and the cylinder with the symmetry boundary are slightly larger than that of the single isolated cylinder. The mean drag force increases with the gap spacing in the case with the wall boundary. As the interference of the wall or symmetry boundary or the other cylinder wake on the cylinder wake becomes weak with the gap spacing increasing, all the drag forces are expected to approach the value of the single isolated cylinder as $e$ is sufficiently large. For the lift force, its magnitude of the upper cylinder decreases with $G$. The lift force magnitudes of the cylinders near the wall boundary are the smallest as $e$ is smaller than 1.5, and the ones of the cylinder near the symmetry boundary are in the middle. With the gap spacing increasing, the lift force trends to the magnitude of the single isolated cylinder. In view of the coefficient approaching the value of the single isolated cylinder, the lift force seems more sensitive to the gap spacing than the drag force.
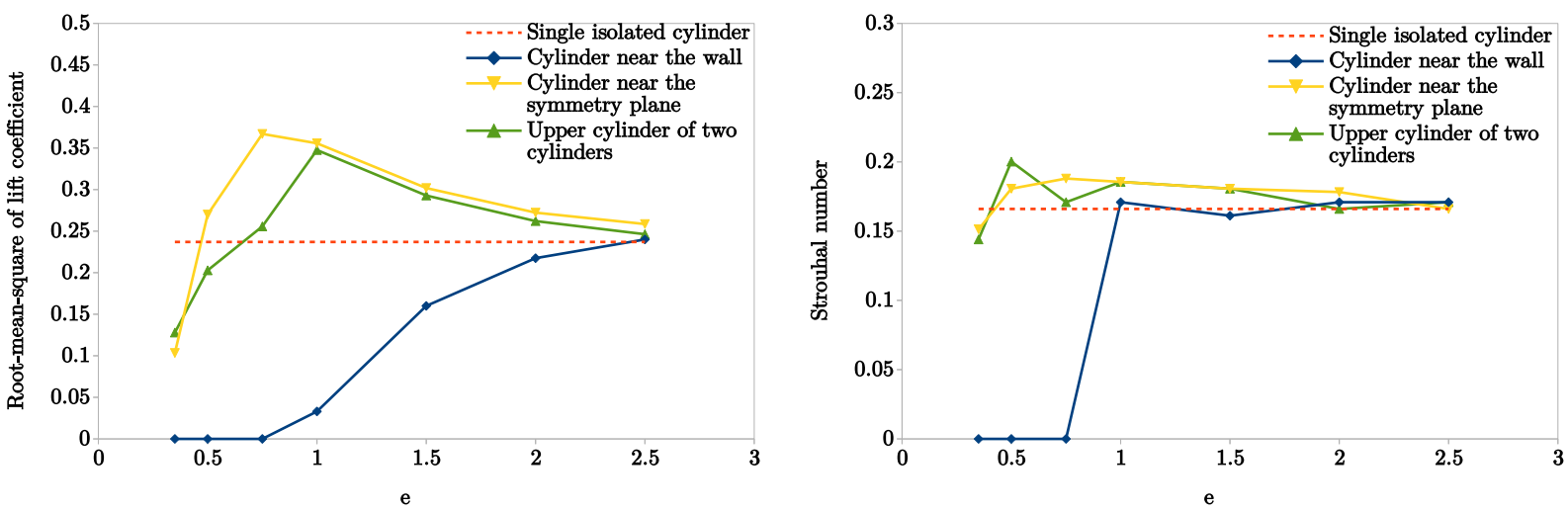

Figure 9: Variations of the RMS of the lift force coefficient and the vortex-shedding frequency (Strouhal numbers) with the gap $e$ (or $G$ ) at $R e=100$.

Figure 9 shows the variation of the RMS of the lift force coefficient and the Strouhal numbers (vortexshedding frequencies) with the gap spacing. In the curves of the RMS, there exists a peak for the two flows with no wall boundary. The RMS reveals that, when the gap spacing is small $(e=0.35$ or $G=0.7)$, the oscillation amplitudes of the lift forces from all the three flows are smaller than that of a single isolated 
cylinder, especially for the flow with the wall boundary, in which the RMS is zero. From $e=0.75$ to 1 , it can been that the RMS magnitude for the cylinder near the wall noticeably increases and is no longer close to zero, which indicates that the stronger vortex shedding takes place between $e=0.7$ and 1 . All the RMS values approach that of a single isolated cylinder as the gap spacing increases. From the curve for the Strouhal numbers, it can be seen that the vortex-shedding frequencies of the two flows with no wall boundary are close to that of a single isolated cylinder. For the flow with the wall boundary, the Strouhal number is zero for $e=0.35,0.5$ and 0.75 as there is no lift force oscillation, and a sharp increase appears in $0.75<e<1$. After that, its value approaches the frequency of a single isolated cylinder.

For all the cases considered in this part, it should be noted that the cylinder is located in the boundary layer $(e+0.5<\delta=2.329)$ when $e \leq 1.5$. Thus the different gap spacing between the cylinder and wall partly reflects the different location in the boundary layer with different thickness. From the obvious variation of the results with the gap spacing, it can be expected that the boundary layer thickness also has strong effect. When $e \geq 2$, the cylinder is outside the boundary layer $(e+0.5>2.329)$, the statistical force coefficients approach those of the single isolated cylinder, which implies that the effect of the boundary shear flow becomes weak.

\subsection{Effect of Reynolds number}

In addition to the effect of the gap spacing, it is also interesting to investigate the effect of the Reynolds number. When the body is near the wall, the Reynolds number has not only direct effect on the flow past cylinder, but also indirect effect through the change of the incoming Blasius flow. In this section, simulations are undertaken over a range of Reynolds numbers $(R e=100,200,300,400,600,800)$ for $e=0.5$. In order to provide a better illustration for the effect of different boundary conditions on the cylinder wake, the hydrodynamic forces of a single isolated cylinder at these Reynolds numbers are presented as well.

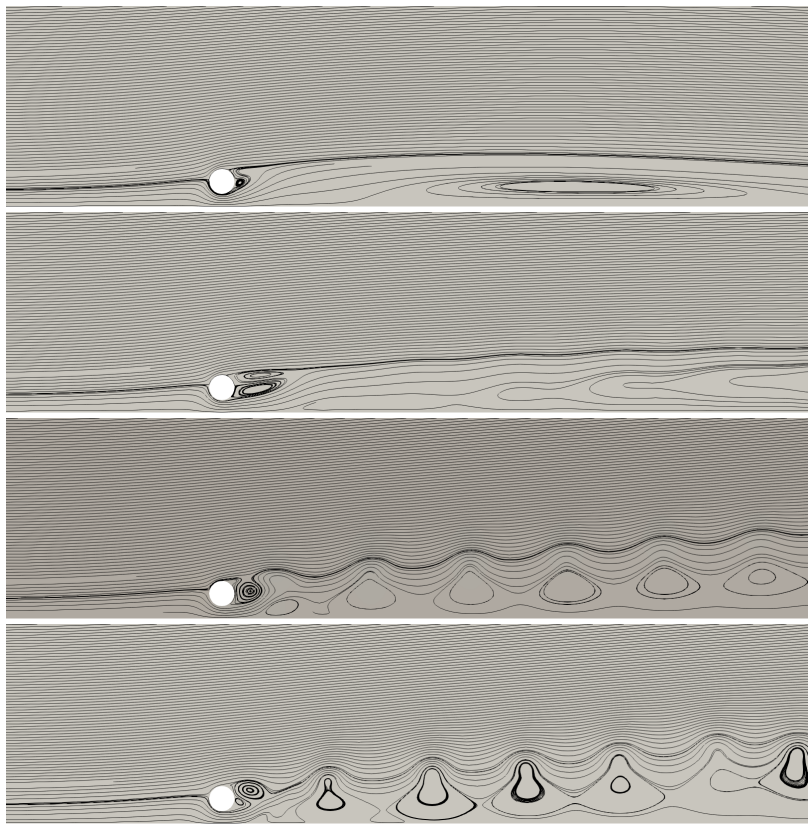

(a)

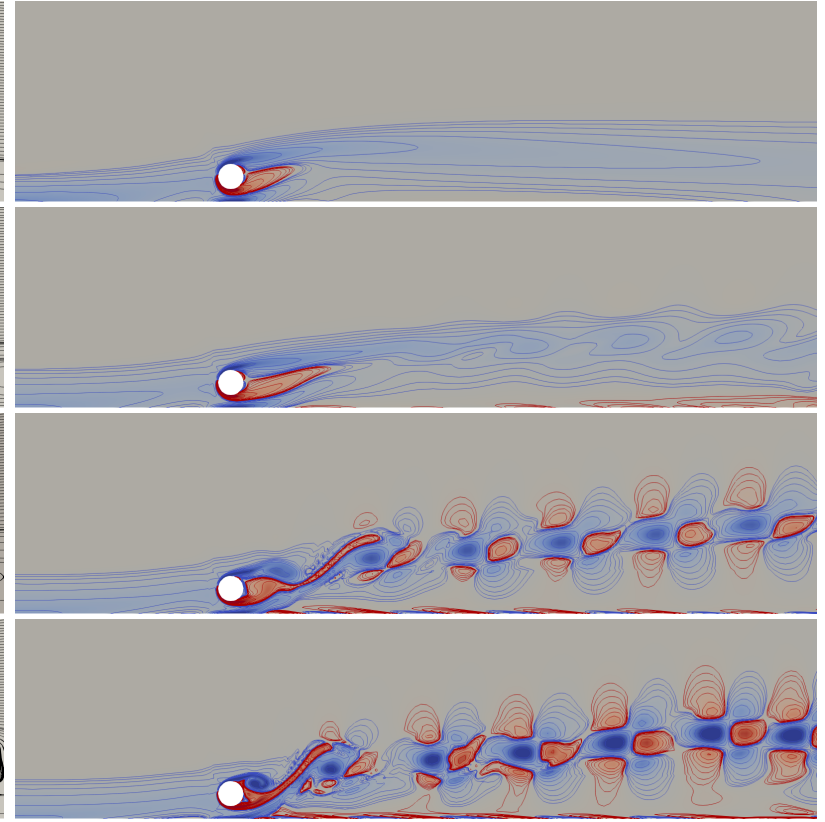

(b)

Figure 10: (a) Streamlines of instantaneous flow, (b) vorticity contours, red colour: positive vorticity, blue colour: negative vorticity, lines correspond to constant $w$ with $|w|<4$. From top to bottom: flow with the wall boundary with $e=0.5$ at $R e=100,200,400$ and 800 . 


\subsubsection{Flow characteristics}

It had been observed in the previous sections that, for the flow with the wall of $e=0.5$ at $R e=100$, the vortex shedding in the wake is suppressed and the wake turns out to be a steady vortex. Figure 10 shows streamline patterns and vorticity contours of instantaneous flows at different $R e$. It is clear that the flow past the cylinder close to the wall boundary is sensitive to the Reynolds number. At $R e=100$ or 200 , the wake is steady after a transition period, and a long vortex street is finally present near the wall in the downstream wake. From the vorticity contours at $R e=100$ or 200 , it can be seen that the vorticities in the downstream wakes are negative, the anti-clockwise vortices (positive vorticity) in the wakes are suppressed by the shear layers with negative vorticity from the wall so that the vortex shedding does not occur. As increases, the vorticity contours clearly display vortices in pair in the wake(with negative and positive vorticity), which implies that the unsteady vortex shedding occurs. At these flows, because of high shear stress of the three shear layers behind the cylinder and near the wall, the paired vortices in the wake result in a series of vortices along the wall, which are clearly shown by the vortical structures in the streamline patterns at $R e=400$ and 800 . Through the vorticity contour, the high magnitude of the vorticity illustrates the strong interaction of the three shear layers at $R e=800$.

\subsubsection{Hydrodynamic forces}
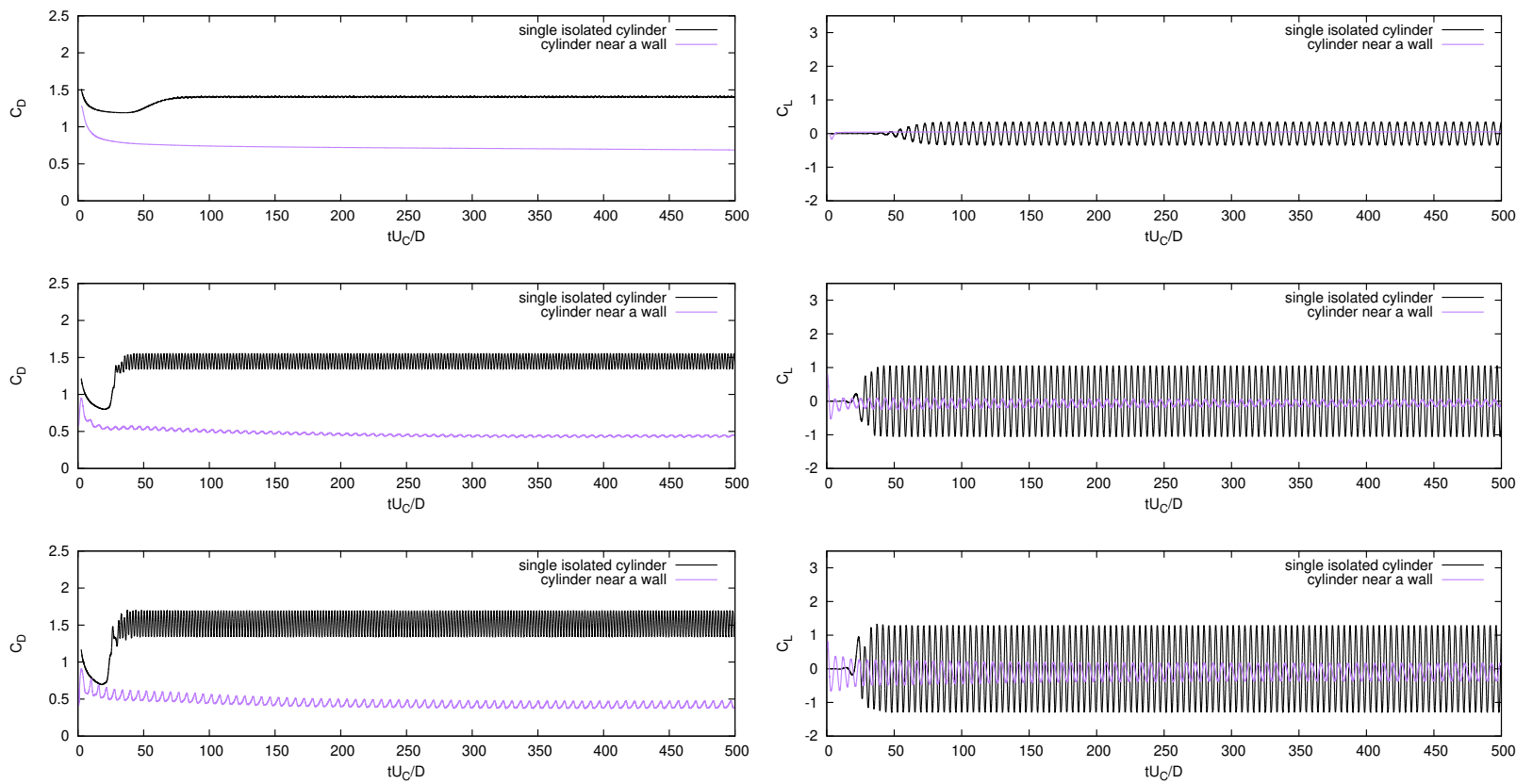

Figure 11: Time history of the drag and lift force coefficients with $e=0.5$ (or $G=1$ ) at $R e=100,400$ and 800 (from top to bottom).

It is of significant practical importance to understand the effect of the $R e$ on the drag and lift force coefficients near the wall. Figure 11 respectively shows the time history of the lift and drag force coefficients for the single isolated and near-wall cylinder at $R e=100,400$ and 800 . It is clear that the curves of the drag and lift forces of the single isolated cylinder at the three Reynolds numbers stay oscillatory, and the oscillation amplitudes of the coefficients increase with the Reynolds number. For the cylinder close to the wall, in an agreement with the flow characteristics, the drag and lift forces remain constant after the flow is fully developed at $R e=100$, which confirms that the unsteady vortex shedding does not occur at this flow. The curves of the two coefficients appear to be oscillatory at $R e=400$ and 800 , and it can be observed that the oscillation amplitude of them increases as Re increases from 400 to 800, which implies that the vortex shedding strengthens as Re increases. 

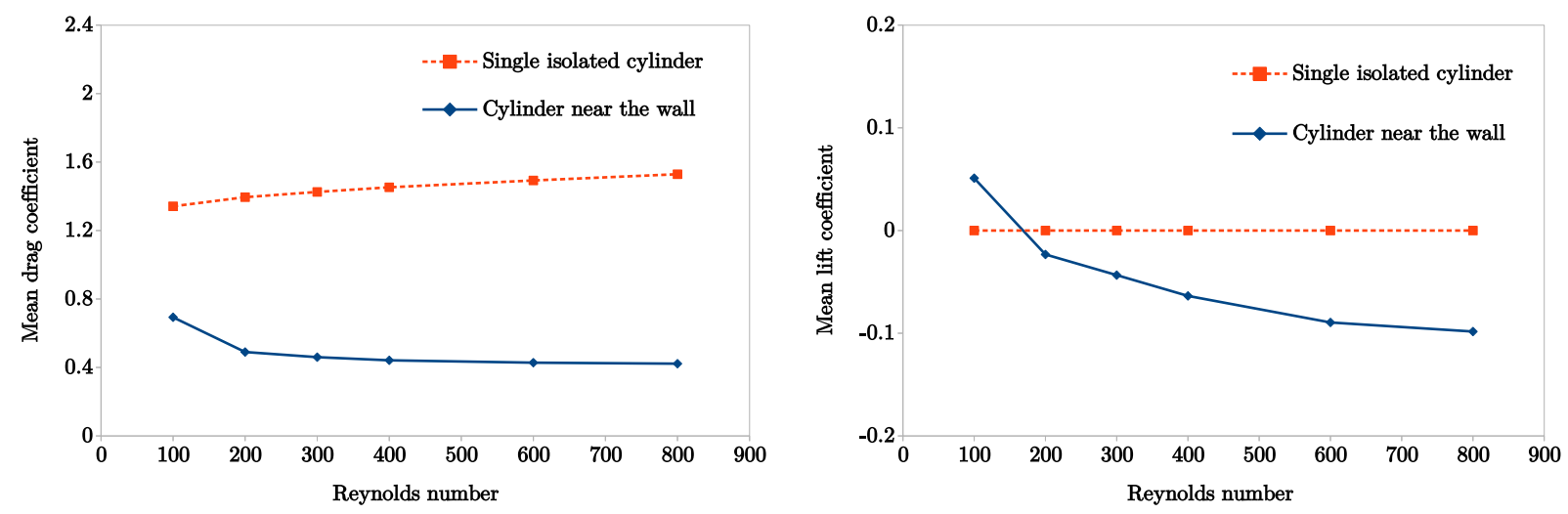

Figure 12: Variation of the mean $\operatorname{drag}\left(\bar{C}_{D}\right)$ and lift $\left(\bar{C}_{L}\right)$ force coefficients with $\operatorname{Re}$ at $e=0.5$ (or $G=1$ ).

Figure 12 shows the mean drag and lift force coefficients at different Reynolds numbers. The mean drag force coefficients of the single isolated cylinder slightly increase with Re. The mean lift coefficients are zero due to the flow symmetry. However, the forces of the near-wall cylinder behave differently. The mean drag forces of the cylinder near the wall boundary at all the Reynolds numbers are smaller than those of the single isolated cylinder. Looking again the vorticity contours in Figure 10 , the strength of the vorticity in the wake of the cylinder near the wall increases as Re increases, while the pressure in the wake decreases. As a result, the mean drag force of the cylinder near the wall boundary decreases when Re increases from $R e=100$ to 300 , and the decrease continues between $R e=300$ and 800 but it is not evidently visible in the figure. With the effect of the shear layer from the wall, the wake of the near-wall cylinder is no longer symmetric, and thus the mean lift forces are non-zero. Through the flow patterns, the pressure underneath the near-wall cylinder decreases when $R e$ increases. Therefore it can be observed that the mean lift force appears to be negative from $R e=200$, and decreases in the range of $R e$ calculated.
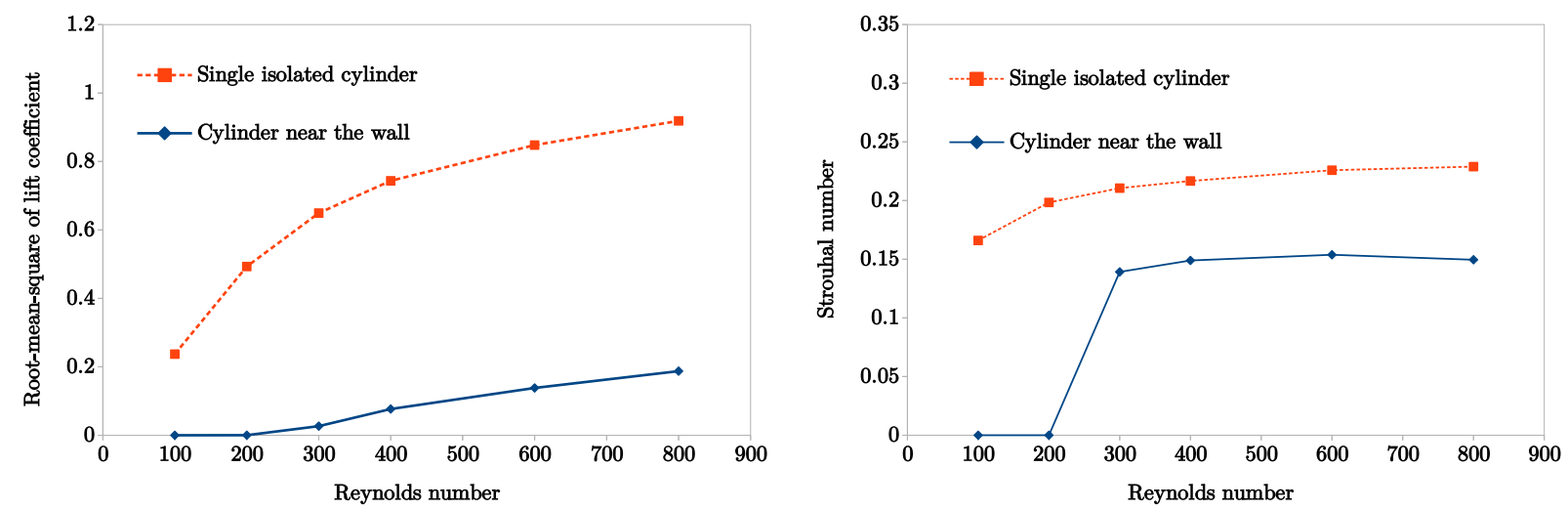

Figure 13: Variations of the RMS of the lift force coefficient and the vortex-shedding frequency (Strouhal numbers) with $R e$ at $e=0.5$ (or $G=1$ ).

Figure 13 shows the variation of the RMS of the lift force coefficient and the Strouhal numbers (vortexshedding frequencies) with Re. It can be seen that, for all the Reynolds numbers, the RMS of the lift force and Strouhal numbers of the near-wall cylinder are smaller than those of the single isolated cylinder, which reveals that the strength of the vortex shedding behind the near-wall cylinder is weaker than that behind the single isolated cylinder and the vortex shedding frequency of the near-wall cylinder is lower than that behind the single isolated cylinder at the same Re, or that the vortex shedding of the near-wall cylinder is 
virtually absent with $C_{L, r m s}=0$ and $S t=0$ at $R e=100$ or 200 . It should be noted that the RMS of the lift force of the single isolated cylinder is sensitive to the Reynolds number, and increases with Re. However, the $C_{L, r m s}$ of the near-wall cylinder is zero at $R e=100$ and 200 , increases to non-zero from $R e=200$ to 300 , continues to increase from $R e=300$. The variation of $C_{L, r m s}$ reveals that, in the flow past the near-wall cylinder with $e=0.5$, the vortex shedding virtually does not occur at $R e=100$ and 200 , and it starts only when $R e$ is about the range of $(200,300)$. The Strouhal numbers of the single isolated cylinder slightly increase with $R e$. For the near-wall cylinder, in accordance with the $C_{L, r m s}$, the Strouhal number stays zero at $R e=100$ and 200 . From $R e=300$ to 800 , it gradually increases slowly.

\subsection{Tabulated results for effects of gap spacing, Reynolds number and boundary layer thickness}

In above two Sections, the effect of the gap spacing at a fixed Reynolds number and the effect of the Reynolds number at a fixed gap spacing on the wake of the near-wall cylinder are shown through various figures. To have some further insight into the effects of gap spacing, Reynolds number and boundary layer thickness, the mean drag $\bar{C}_{D}$, mean lift $\bar{C}_{L}$, RMS of lift $C_{L, r m s}$ and Strouhal number at different gap

Table 4: Mean drag force coefficient of the near-wall cylinder at different gap spacings, Reynolds numbers/boundary layer thicknesses.

\begin{tabular}{|c|c|c|c|c|c|c|c|c|}
\hline \multicolumn{2}{|c|}{$\delta$ e } & & & & & & & \\
& $R e V$ & & & & & & \\
\multicolumn{2}{|c|}{$R$} & & & & & & & \\
\hline 100 & 2.329 & 0.649 & 0.693 & 0.8 & 0.97 & 1.217 & 1.3276 & 1.37 \\
\hline 200 & 2.05 & 0.4742 & 0.49 & 0.7675 & 0.9571 & 1.2011 & 1.3083 & 1.3448 \\
\hline 300 & 1.95 & 0.4212 & 0.46 & 0.7725 & 0.9818 & 1.236 & 1.34 & 1.3707 \\
\hline 400 & 1.899 & 0.3874 & 0.4416 & 0.7884 & 1.0045 & 1.2674 & 1.3664 & 1.3937 \\
\hline 600 & 1.845 & 0.423 & 0.4278 & 0.8081 & 1.036 & 1.3034 & 1.3997 & 1.4243 \\
\hline 800 & 1.8177 & 0.42 & 0.422 & 0.8168 & 1.0615 & 1.33 & 1.429 & 1.452 \\
\hline
\end{tabular}

Table 5: Mean lift force coefficient of the near-wall cylinder at different gap spacings, Reynolds numbers/boundary layer thicknesses.

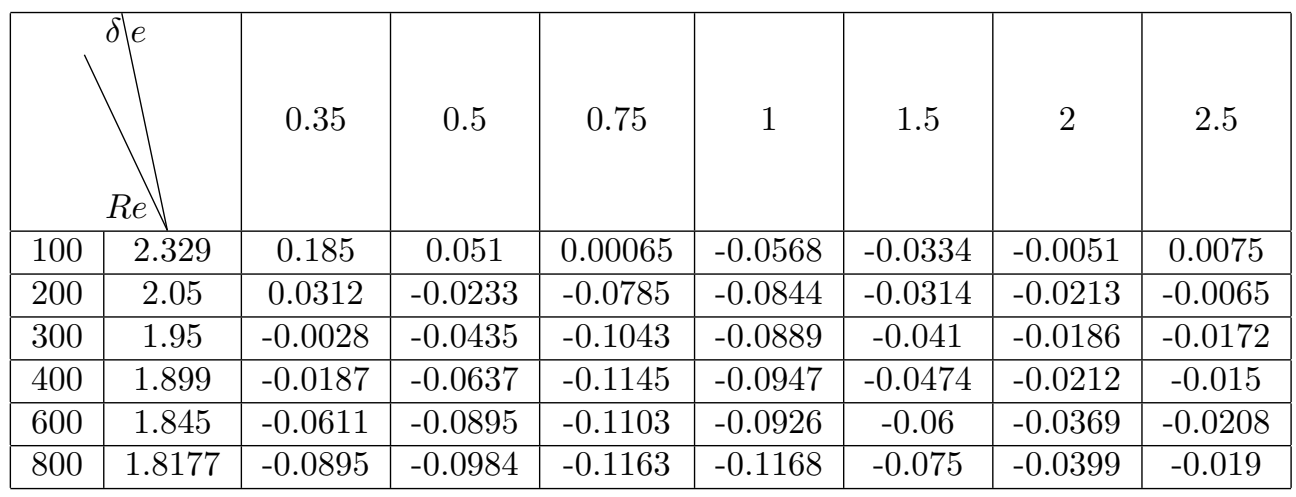

spacings, Reynolds numbers and boundary layer thicknesses are provided in the following tables. As the location of the cylinder is fixed, at each given Reynolds number, the boundary layer thickness is also fixed.

It can be seen from Tables 4 and 5 that, for the fixed Reynolds numbers or boundary layer thicknesses, the mean drag increases with the gap spacing, the mean lift decreases first, reaches a trough, and then 
increases. When the gap spacing is fixed, the mean drag varies differently with the Reynolds number, and the mean lift decreases with the Reynolds number.

In a similar way, the RMS of lift force coefficient and Strouhal number are given in Table 6 and 7 . It has been mentioned previously that vortex shedding is very weak when $e \leq 0.75$ at $R e=100$ or $e \leq 0.5$ at $R e=200$ or $e=0.35$ at $R e=300$. For all the cases in which the vortex shedding is no longer weaker, the RMS of lift force coefficients increases with the gap spacing. On the other hand, it increases with the Reynolds number when the gap spacing is fixed. The vortex shedding frequency increases with the Reynolds number except in the case of $R e=200$. At a fixed Reynolds number, the vortex shedding frequency increases with the gap spacing.

Table 6: RMS of lift force coefficient of the near-wall cylinder at different gap spacings, Reynolds numbers/ boundary layer thicknesses.

\begin{tabular}{|c|c|c|c|c|c|c|c|c|}
\hline \multicolumn{2}{|c|}{$\delta(e)$} & & & & & & & \\
\multicolumn{2}{|c|}{$R e$} & & & & & & & \\
& & & & & & & & \\
\hline 200 & 2.329 & 0 & 0 & 0 & 0.033 & 0.16 & 0.2175 & 0.24 \\
\hline 300 & 2.05 & 0 & 0 & 0.1103 & 0.2393 & 0.411 & 0.478 & 0.501 \\
\hline 400 & 1.95 & 0 & 0.0266 & 0.2264 & 0.4063 & 0.5862 & 0.6515 & 0.6685 \\
\hline 600 & 1.899 & 0.0052 & 0.0766 & 0.3347 & 0.5169 & 0.6989 & 0.7616 & 0.7753 \\
\hline 800 & 1.8177 & 0.0777 & 0.1382 & 0.4578 & 0.6364 & 0.8219 & 0.8837 & 0.8949 \\
\hline
\end{tabular}

Table 7: Strouhal number of the near-wall cylinder at different gap spacings, Reynolds numbers/boundary layer thicknesses.

\begin{tabular}{|c|c|c|c|c|c|c|c|c|}
\hline \multicolumn{2}{|c|}{$\delta(e$} & & & & & & & \\
\multicolumn{2}{|c|}{$R e$} & & & & & & & \\
& & & & & & & \\
\hline 100 & 2.329 & 0 & 0 & 0 & 0.1709 & 0.1611 & 0.1709 & 0.1709 \\
\hline 200 & 2.05 & 0 & 0 & 0.1611 & 0.1758 & 0.1312 & 0.1373 & 0.1404 \\
\hline 300 & 1.95 & 0 & 0.1392 & 0.1758 & 0.1868 & 0.2051 & 0.2136 & 0.2136 \\
\hline 400 & 1.899 & 0.1416 & 0.1489 & 0.1807 & 0.1904 & 0.2148 & 0.2197 & 0.2246 \\
\hline 600 & 1.845 & 0.1648 & 0.1538 & 0.1840 & 0.2005 & 0.2225 & 0.2307 & 0.2307 \\
\hline 800 & 1.8177 & 0.1648 & 0.1495 & 0.1892 & 0.2075 & 0.2288 & 0.235 & 0.2383 \\
\hline
\end{tabular}

\section{Conclusions}

An investigation on boundary shear flow past a circular cylinder near a wall has been numerically performed, in which a stabilized finite element method and a generalized- $\alpha$ method are applied to space discretization and time integration of the impressible Navier-Stokes equations respectively. The investigation has shed some light into the major difference between the boundary layer flow past a circular cylinder near a wall and the flows corresponding to the symmetry boundary, and to two identical circular cylinders in a side-by-side arrangement. Through the obtained results, we can draw the following conclusions. 
1. At $R e=100$, with the effect of the boundary layer flow, the vortex contour lines near the wall in the wake of the cylinder shift upwards. With a reduction of the gap spacing, the lower shear layer behind the cylinder is suppressed by the shear layer from the boundary layer flow near the wall, partly due to lower effective Reynolds number, resulting in a steady state in the wake.

2. At $R e=100$, the $\bar{C}_{D}, C_{L, r m s}$ and $S t$ number of the circular cylinder near the wall are more sensitive to the gap spacing in contrast with those in the other two flows. When the cylinder locates in the boundary layer, the boundary shear flow has strong effect on the hydrodynamic quantities of the cylinder.

3. For a fixed gap between the cylinder and the wall $(e=0.5)$, the flow past the near-wall cylinder is steady at low Reynolds numbers. The periodic vortex shedding occurs with a series of vortices present along the wall as $R e$ increases.

4. At $e=0.5$, the mean lift force and RMS of the lift force of the near-wall cylinder are sensitive to $R e$. The mean lift force decreases with $R e$. The $C_{L, r m s}$ is virtually zero when Reynolds number is around $R e=100$ to 200 and then increases with $R e$. The mean drag force and Strouhal number are not much sensitive to $R e$ after the vortex shedding starts from $\operatorname{Re}=300 . R e=300$.

As a conclusion, the boundary shear flow has strong effect on the flow and forces of the cylinder, not only through the variation of gap spacing but also the Reynolds number. In the boundary layer flow at $R e=100$, the vortex shedding from the cylinder is suppressed below a certain $e$ in $(0.75,1)$. At a certain gap spacing, there is a certain Reynolds number at which the vortex shedding of the cylinder in the boundary layer flow become significant. For the example of $e=0.5$, it is found in the present study that the vortex shedding starts above a $R e$ in $(200,300)$.

\section{Acknowledgments}

The authors wish to express our appreciation for the support provided by the Natural Science Foundation (Grant No. SBK2018040999) funded by Jiangsu Science, Technology Office and the General Foundation (Grant No. 18KJB570001) funded by Jiangsu Education and the National Natural Science Foundation of China (Grant No. 51879123).

\section{References}

[1] M. M. Zdravkovich, Review of flow interference between two circular cylinders in various arrangements, Journal of Fluids Engineering 99 (4) (1977) 618-633.

[2] D. Sumner, S. S. T. Wong, S. J. Price, M. P. Padoussis, Fluid behaviour of side-by-side circular cylinders in steady cross-flow, Journal of Fluids and Structures 13 (3) (1999) 309-338.

[3] C. H. K. Williamson, Evolution of a single wake behind a pair of bluff bodies, Journal of Fluid Mechanics 159 (337) (1985) $1-18$.

[4] S. Kang, Characteristics of flow over two circular cylinders in a side-by-side arrangement at low reynolds numbers, Physics of Fluids 15 (9) (2003) 2486-2498.

[5] H. J. Kim, P. A. Durbin, Investigation of the flow between a pair of circular cylinders in the flopping regime, Journal of Fluid Mechanics 196 (196) (1988) 431-448.

355 [6] S. J. Xu, Y. Zhou, R. M. C. So, Reynolds number effects on the flow structure behind two side-by-side cylinders, Physics of Fluids 15 (5) (2003) 1214-1219.

[7] H. Jiao, G. Wu, Analysis of fluctuating force acting on two cylinders in different arrangements through lattice boltzmann method, Journal of Fluids and Structures 82 (20) (2018) 101-120.

[8] P. W. Bearman, M. M. Zdravkovich, Flow around a circular cylinder near a plane boundary, Journal of Fluid Mechanics 89 (1) (1978) 33-47.

[9] M. M. Zdravkovich, Forces on a circular cylinder near a plane wall, Applied Ocean Research 7 (4) (1985) $197-201$.

[10] G. Buresti, A. Lanciotti, Vortex shedding from smooth and roughened cylinders in cross-flow near a plane surface, Aeronaut Q 30 (1979) 305-321.

[11] A. J. Grass, J. A. Bray, P. W. J. Raven, R. J. Stuart, The influence of boundary layer velocity gradients and bed proximity on vortex shedding from free spanning pipelines, Journal of Energy Resources Technology 106 (1) (1983) 70-78.

[12] S. Taniguchi, K. Miyakoshi, Fluctuating fluid forces acting on a circular cylinder and interference with a plane wall, Experiments in Fluids 9 (4) (1990) 197-204.

[13] C. Lei, L. Cheng, K. Kavanagh, Re-examination of the effect of a plane boundary on force and vortex shedding of a circular cylinder, Journal of Wind Engineering and Industrial Aerodynamics 80 (3) (1999) 263-286. 
[14] S. Prince, D. Summer, J. Smith, K. Leong, M. Padoussis, Flow visualization around a circular cylinder near to a plane wall, Journal of Fluids and Structures 16 (2) (2002) 175-191.

[15] X. K. Wang, S. K. Tan, Near-wake flow characteristics of a circular cylinder close to a wall, Journal of Fluids and Structures 24 (5) (2008) 605-627.

[16] S. Taneda, Experimental investigation of vortex streets, Tokyo Sugaku Kaisya Zasshi 20 (9) (2013) $1714-1721$.

375 [17] M. C. Ong, T. Utnes, L. E. Holmedal, D. Myrhaug, B. Pettersen, Numerical simulation of flow around a circular cylinder close to a flat seabed at high reynolds numbers using a k- $\epsilon$ model, Coastal Engineering 57 (10) (2010) 931-947.

[18] C. Lei, L. Cheng, S. W. Armfield, K. Kavanagh, Vortex shedding suppression for flow over a circular cylinder near a plane boundary, Ocean Engineering 27 (10) (2000) 1109-1127.

[19] T. J. Hughes, Multiscale phenomena : Green's functions, the dirichlet-to-neumann formulation, subgrid scale models, bubbles and the origins of stabilized methods, Computer Methods in Applied Mechanics and Engineering 127 (1-4) (1995) $387-401$.

[20] Y. Bazilevs, V. M. Calo, J. A. Cottrell, T. J. R. Hughes, A. Reali, G. Scovazzi, Variational multiscale residual-based turbulence modeling for large eddy simulation of incompressible flows, Computer Methods in Applied Mechanics and Engineering 197 (1-4) (2007) 173-201.

[21] L. F. Chen, Aspects of pod-based wall-layer modeling for the variational multiscale method., Ph.D. thesis, Delft, The Netherlands (2015).

[22] K. E. Jansen, C. H. Whiting, G. M. Hulbert, A generalized-alpha method for integrating the filtered navier-stokes equations with a stabilized finite element method, Computer Methods in Applied Mechanics and Engineering 190 (3-4) (2000) 305319.

23] H. K. W. C, Three-dimensional wake transition, Journal of Fluid Mechanics 328 (1996) 345-407.

[24] M. A. Prsic, M. C. Ong, B. Pettersen, D. Myrhaug, Large eddy simulations of flow around a circular cylinder close to a flat seabed, Marine Structures 46 (2016) 127-148.

[25] F. Shakib, Finite element analysis of the compressible euler and navier-stokes equations, Ph.D. thesis (1989).

[26] C. A. Taylor, T. J. R. Hughes, C. K. Zarins, Finite element modeling of blood flow in arteries, Computer Methods in Applied Mechanics and Engineering 158 (158) (1998) 155-196.

[27] Y. Bazilevs, C. Michler, V. M. Calo, T. J. R. Hughes, Isogeometric variational multiscale modeling of wall-bounded turbulent flows with weakly enforced boundary conditions on unstretched meshes, Computer Methods in Applied Mechanics and Engineering 199 (13-16) (2010) 780-790.

[28] L. F. Chen, X. Q. Hu, A pod-based variational multiscale method for large eddy simulation of turbulent channel flows, International Journal of Computational Methods 14 (5) (2017) 1750049.

[29] G. X. Wu, Z. Z. Hu, A taylor series-based finite volume method for the naviercstokes equations, International Journal for Numerical Methods in Fluids 58 (12) (2008) 1299-1325.

[30] P. K. Kundu, I. M. Cohen, D. R. Dowling, Fluid Mechanics (Fifth Edition), 2012.

[31] J. Park, K. Kwon, H. Choi, Numerical solutions of flow past a circular cylinder at reynolds numbers up to 160, KSME International Journal 12 (6) (1998) 1200-1205.

[32] A. Placzek, J. F. Sigrist, A. Hamdouni, Numerical simulation of vortex shedding past a circular cylinder in a cross-flow at low reynolds number with finite volume-technique: Part 1 - forced oscillations, in: ASME Pressure Vessel and Piping Division Conference, 2007, pp. 11-20. 